\title{
Notes On Acceptance/Rejection/Delay of Manuscripts \\ Submitted to Weed Technology
}

A smooth, orderly, and efficient flow of manuscripts through the reviewing and editing process is in the best interests of all concerned. Some manuscripts are received in excellent condition; others require further attention in order to meet acceptable standards. Authors can do much to expedite publication of their results by submitting carefully prepared, scientifically sound manuscripts initially.

Rarely does a manuscript that is inordinately delayed or eventually deemed to be unacceptable have only a single deficiency. Listed below are some of the more common reasons for rejection, major revision, withdrawal, referral, and/or significant delay of manuscripts sent to Weed Technology.

1. Subject too far afield or better suited for another journal.

- Manuscript with little or no reference to weeds, herbicides, weed science, or weed technology.

- Manuscripts dealing exclusively with synthetic plant growth regulators or pesticides that are not listed or recognized as herbicides applied to crops, i.e., agronomic or horticultural studies but not weed technology.

- Purely "opinion" papers (not Presidential or Honorary Member addresses), which would be more appropriate in an editorial column, trade magazine, letters to the editor, forum, or lobbying.

2. Experiments poorly designed and/or conducted. Inadequate controls, replications, repetitions, etc. Major data lost to weather, animals, etc. but work not repeated.

3. Insufficient new information to warrant publication; makes no significant, new, useful contribution to knowledge.

4. Experiments not repeated in time or space. Some manu- scripts based on a single greenhouse experiment. (Rare exceptions are permitted in the NOTES section, with proper justification.)

5. Incomplete research-additional studies needed, manuscripts more appropriate as progress reports for regional conference proceedings, "promissory notes" rather than "finished" research.

6. Manuscripts poorly written, also apparently never having been subjected to internal review before submission.

7. Improper use of statistics, faulty interpretation of data, statements made in Results and Discussion that disagree with the actual data in tables and figures. Inadequate attention to such details.

8. Manuscripts not prepared in accordance with Directions for Contributors to Weed Technology. (Also, insufficient copies provided, no stick-on labels or suggested reviewers. Note: These are more nuisance factors and a cause of delays and extra expense rather than a reason for rejection, of course.)

9. Insufficient actual data-e.g., one manuscript had no quantitative measurements, but observations only.

10. Manuscript purely descriptive, not interpretive or showing application to weed technology-e.g., micrographs of plants without significant connection to weed technology.

11. Manuscript overlaps significantly with paper(s) either previously published or submitted concurrently. In the latter instances, manuscripts are sometimes combined. Research papers that are known to have been published elsewhere are not accepted.

12. Some multiple-author papers were not reviewed and edited carefully by all authors! 